\title{
Improvements in glucose tolerance and insulin sensitivity after lifestyle intervention are related to changes in serum fatty acid profile and desaturase activities: the SLIM study
}

\author{
E. Corpeleijn • E. J. M. Feskens • E. H. J. M. Jansen • \\ M. Mensink • W. H. M. Saris • T. W. A. de Bruin • \\ E. E. Blaak
}

Received: 26 April 2006 / Accepted: 26 June 2006 / Published online: 3 August 2006

(C) Springer-Verlag 2006

\begin{abstract}
Aims/hypothesis The aim of this study was to investigate whether lifestyle intervention-induced changes in serum fatty acid profile of cholesteryl esters and estimated desaturase activities are related to improvements in insulin sensitivity in subjects at risk of type 2 diabetes.

Materials and methods In the Study on Lifestyle Intervention and Impaired Glucose Tolerance Maastricht (SLIM), 97 men

E. Corpeleijn • M. Mensink - W. H. M. Saris • E. E. Blaak Department of Human Biology, The Nutrition and Toxicology

Research Institute NUTRIM, Maastricht University,

Maastricht, The Netherlands

E. J. M. Feskens

Centre for Nutrition and Health,

National Institute for Public Health and the Environment,

Bilthoven, The Netherlands

E. J. M. Feskens

Division of Human Nutrition,

Wageningen University and Research Center,

Wageningen, The Netherlands

E. H. J. M. Jansen

Laboratory for Toxicology, Pathology and Genetics,

National Institute for Public Health and the Environment,

Bilthoven, The Netherlands

T. W. A. de Bruin

Department of Medicine and Endocrinology,

Cardiovascular Research Institute CARIM,

University Hospital Maastricht,

Maastricht, The Netherlands

E. Corpeleijn $(\triangle)$

Department of Human Biology, Faculty of Health Sciences,

Maastricht University,

P.O. Box 616, 6200 Maastricht, MD, The Netherlands

e-mail: E.Corpeleijn@hb.unimaas.nl
\end{abstract}

and women with IGT were randomised to a combined diet and exercise programme (47 intervention) or a control group (50 control subjects). At baseline and after 1 year the following assessments were made: an OGTT, an exercise test to determine maximal aerobic capacity, anthropometry, and analysis of the serum fatty acid profile of cholesteryl esters.

Results The lifestyle programme was effective in reducing the intake of total and saturated fat, increasing physical activity, reducing obesity and improving insulin sensitivity and glucose tolerance. Regression analysis of the total population showed that an increase in the C20:4 n-6/C20:3 $n$-6 ratio (estimated $\Delta 5$-desaturase activity) and reductions in the C18:3 n-6/C18:2 $n-6$ ratio (estimated $\Delta 6$-desaturase activity) and the C16:1 n-7/C16:0 ratio (estimated $\Delta 9$ desaturase activity or stearoyl-CoA desaturase-1) were significantly associated with a decrease in homeostasis model assessment for insulin resistance. After adjustment for lifestyle changes (change in percentage body fat, aerobic capacity and saturated fat intake), these associations were partly reduced, but remained statistically significant. Conclusions/interpretation Lifestyle-induced changes in fatty acid profile of cholesteryl esters and desaturase activities were independently related to changes in insulin sensitivity in subjects at risk of type 2 diabetes.

Keywords Diet - Exercise - Fat intake .

Impaired glucose tolerance · Insulin resistance - Obesity · Saturated fat $\cdot$ Serum fatty acid composition . Stearoyl-CoA desaturase- $1 \cdot$ Unsaturated fat
Abbreviations
$E \% \quad$ energy \%
HOMA- homeostasis model assessment for insulin
IR resistance 
MUFA monounsaturated fatty acids

PUFA polyunsaturated fatty acids

SCD-1 stearoyl-CoA desaturase-1

SFA saturated fatty acids

$\mathrm{VO}_{2 \max }$ maximal oxygen consumption

\section{Introduction}

Interest in the fat quality of the Western diet has previously been generated by the results of cross-sectional epidemiological studies. These showed that the consumption of saturated fat was inversely related to insulin sensitivity and glucose tolerance, whereas a positive association was found for unsaturated fat [1-3]. Similar results were found for fatty acid composition in serum [4, 5]. In addition, prospective studies in healthy subjects have shown that a serum fatty acid profile of high saturated fatty acids or low unsaturated fatty acids can predict the development of type 2 diabetes [1, 6, 7]. Two well-controlled human studies recently demonstrated that replacing saturated fatty acids (SFA) in the diet with either monounsaturated fatty acids (MUFA) [8] or polyunsaturated fatty acids (PUFA) [9] resulted in changes in serum fatty acid profile and improved insulin sensitivity. This improvement in insulin sensitivity was found particularly in subjects with a relatively low total fat intake (below median 37 energy \% [E\%]) [8].

The plasma fatty acid profile is influenced not only by dietary fat intake, but also by endogenous fatty acid metabolism, e.g. by desaturase enzymes. An important role of the desaturase enzymes is regulation of the degree of unsaturation of lipids throughout the body. This is important for the fluidity of cell membranes, affecting cell permeability and signalling, including insulin signalling [10]. The $\Delta 9$-desaturase catalyses the conversion of palmitic and stearic acid into palmitoleic acid and oleic acid, respectively. These MUFA are required for membrane phospholipids and the synthesis of adipose tissue triglycerides and cholesteryl esters $[10,11]$. The $\Delta 5$ - and $\Delta 6$ desaturases catalyse the synthesis of $n-6$ and n-3 PUFA [10], of which, arachidonic acid (C20:4 n-6) is an eicosanoid precursor, and docosahexaenoic acid (C22:6 n-3) plays a role in the function of the retina and central nervous system. Some specific highly unsaturated fatty acids are regulators of the expression of genes involved in lipogenesis and lipid oxidation [12]. The activity of desaturase enzymes has been associated with insulin resistance [13-15]. Animal studies have shown that mice lacking stearoyl-CoA desaturase-1 (SCD-1), a mouse isoform of $\Delta$ 9-desaturase, are more insulin sensitive than their wildtype littermates [13]. In human studies, an increased $\Delta 6$-desaturase activity and a decreased $\Delta 5$-desaturase activity have been associated with insulin resistance and type 2 diabetes [14, 16, 17]. Recently, a 20-year prospective study in healthy Swedish men showed that high estimated $\Delta 9$ - and $\Delta 6$-desaturase activities and low $\Delta 5$-desaturase activity predicted the development of the metabolic syndrome [18]. These studies suggest that desaturase enzymes may be directly or indirectly involved in the development of insulin resistance.

There is increasing evidence that serum fatty acid profiles and fatty acid desaturase activities may be influenced by lifestyle factors such as diet [3, 16] and exercise $[19,20]$. We have previously reported that a combined lifestyle intervention programme was effective in improving glucose tolerance and insulin sensitivity in prediabetic subjects, thereby reducing the risk of diabetes [21]. Based on these findings, the aim of this study was to investigate whether changes in the fatty acid profile of serum cholesteryl esters and estimated fatty acid desaturase activities were related to lifestyle intervention-induced changes in glucose tolerance and insulin sensitivity.

\section{Subjects and methods}

\section{Subjects}

Subjects were recruited from a large existing cohort of the general population [22] and through advertisements in the local newspaper. Subjects were screened for IGT with a standard OGTT with capillary sampling according to the World Health Organization guidelines [23]. Subjects with IGT were invited to undergo a second OGTT and were included when the mean 2 -h glucose concentration was between 7.8 and $12.5 \mathrm{mmol} / \mathrm{l}$. Exclusion criteria were previously diagnosed diabetes other than gestational diabetes, use of medication known to interfere with glucose metabolism, participation in vigorous exercise or an intensive weight loss programme in the year prior to participation, and any (chronic) disease that makes participation in a lifestyle programme impossible or has an improbable 5-year survival. The study protocol was approved by the Medical Ethics Committee of Maastricht University. All subjects gave written informed consent.

Subjects were randomized to the intervention group or the control group, with stratification for sex and 2-h glucose value. Of the 147 subjects, 131 completed the 1-year intervention period. We obtained complete datasets of general and metabolic characteristics and serum fatty acid profile of cholesteryl esters in 97 subjects for regression analysis. Incomplete datasets were mainly due to missing values for dietary intake and aerobic capacity (maximal oxygen consumption $\left.\left[\mathrm{VO}_{2 \max }\right]\right)$. There was a higher proportion of women in the group of excluded subjects 
$(+29 \%)$, but there were no differences in age, BMI, 2-h glucose values or homeostasis model assessment for insulin resistance (HOMA-IR) values between excluded and included subjects. There were a comparable number of excluded subjects in the intervention $(n=27)$ and control $(n=23)$ groups. The results of this lifestyle intervention have been published previously [21].

\section{Lifestyle intervention programme}

The lifestyle intervention programme comprised a dietary and physical activity part. Dietary recommendations were based on the Dutch guidelines for a healthy diet [24] and consisted of a carbohydrate intake of at least $55 \mathrm{E} \%$; a total fat intake below 30-35E\%, with a saturated fat intake below $10 E \%$ and a cholesterol intake $<33 \mathrm{mg} / \mathrm{MJ}$. No (very)-low-calorie diets were used. Dietary advice was given by a skilled dietician, on an individual basis, after consideration of an individual 3-day weighed food record. The first visit took place 4-6 weeks after randomisation. A visit was scheduled every 3 months thereafter. Subjects were encouraged to increase their physical activity to at least $30 \mathrm{~min}$ of moderate physical activity a day for at least 5 days a week [25]. At the start of the study, individual advice was given on how to increase daily physical activity (walking, cycling, swimming), and well-defined goals were set. Furthermore, subjects were encouraged to participate in an exercise programme, especially designed for this study, with both aerobic exercise training and resistance training components. Exercise sessions were supervised by trainers. Subjects had free access to these training sessions and were advised to participate for at least $1 \mathrm{~h}$ a week. Once every year, subjects in the control group received oral and written information about the beneficial effects of a healthy diet, weight loss and increased physical activity; no individual advice or programmes were provided.

\section{Measurements}

Glucose tolerance was monitored with a standard OGTT with venous blood sampling at baseline and at $2 \mathrm{~h}$ [22]. During the same visit, body weight was measured to the nearest $0.1 \mathrm{~kg}$ on an electronic scale, with the subject wearing only light clothing. Height was measured to the nearest $0.5 \mathrm{~cm}$ without shoes. Skinfold thickness was measured twice using a skinfold caliper at the triceps, biceps, subscapular and suprailiacal regions. The sum of skinfolds was used to calculate body fat percentage [26]. Waist circumference (waist) was measured with the subject in standing position at the level midway between the lowest rib and iliac crest to the nearest $0.5 \mathrm{~cm}$. A 3-day weighed food record (two week days and one weekend day) was kept in the 2 weeks before the visit. Food records were checked by a dietician, and the intake of nutrients was calculated with a validated computer program using the Dutch food table (NEVO). An incremental exhaustive exercise test was performed on an electronically braked bicycle ergometer to determine the maximal power output (watts) and maximal oxygen consumption $\left(\mathrm{VO}_{2 \max }\right)$.

Biochemical analysis and calculations

Plasma glucose was measured with a standard enzymatic technique automated on the Cobas Fara centrifugal analyser (Glucose HK 125; ABX Diagnostics, Montpellier, France). Plasma insulin was measured with an ELISA assay (Mercodia, Uppsala, Sweden) with no cross-reactivity with pro-insulin. The HOMA-IR was calculated as described by Matthews et al. [27]. The HOMA index is the outcome of a mathematical model based on dose-response curves for glucose uptake and insulin production in a fasting, steadystate condition. Although it is not suitable for use at the individual level, as is the hyperinsulinaemic-euglycaemic clamp, it is a fairly good marker for insulin resistance in larger groups $(n>30)$ and is a better marker of insulin resistance than fasting insulin, particularly in obese and/or IGT subjects [28]. Glycated haemoglobin $\left(\mathrm{HbA}_{1 \mathrm{c}}\right)$ was determined in fasting serum by HPLC (reference values for our laboratory $4.4-6.2 \%$ ).

The fatty acid profile of serum cholesteryl esters was determined by gas chromatography-flame ionisation detection after solid-phase extraction using a method adapted from that of Agren and coworkers [29]. Shortly after deproteination and chloroform extraction the lipid extract was applied to an aminopropyl solid-phase column (Bond-Elut NH2 $200 \mathrm{mg}$; Varian Ass, Middelburg, the Netherlands) and the cholesteryl-bound fatty acids were eluted with hexane. After hydrolysis and methylation, the fatty acid methyl esters (FAME) were separated on a $100-\times 0.25$-mm ID WCOTfused silica capillary column using a GC-3900 gas chromatograph (Varian Ass). Galaxie software (Varian Ass) was used for quantification and identification of peaks. The relative amount of each fatty acid (percentage of total fatty acids) was quantified by integrating the area under the peak and dividing the result by the total area of all fatty acids. The activity of $\Delta 5$-desaturase was estimated as the product/ precursor ratio (i.e. the proportion of arachidonic acid (C20:4 $n$-6) to dihomo- $\gamma$-linolenic acid [C20:3 $n$-6]), $\Delta 6$-desaturase activity was estimated as the proportion of $\gamma$-linolenic acid (C18:3n-6) to linoleic acid (C18:2 $n$-6), and $\Delta$ 9-desaturase activity was estimated as the proportion palmitoleic acid (C16:1 n-7) to palmitic acid (C16:0).

\section{Statistical analysis}

Correlations were tested using Pearson's correlation coefficient $(r)$, two-tailed. ANOVA for repeated measures was used 
to test differences between groups and changes over time. Regression analysis was performed to identify the contribution of changes in lifestyle and fatty acid profile to changes in insulin resistance (HOMA-IR). When testing a 1-year change as the dependent variable, the mean of the dependent variable ([year $0+$ year 1]/2) was included in the model to correct for regression to the mean. Regression analysis was also performed with adjustment for the means of all variables, but this did not add to the explained variance of the model and did not change the outcomes, so only the mean of the dependent variable was included. All 1-year changes were calculated as year 1-year 0 . To investigate whether the relationship between changes in desaturase activity and changes in insulin resistance was dependent on changes in lifestyle, we tested the contribution of desaturase activity alone (model 1), adjusted for the mean of the dependent variable. We further adjusted for the intake of SFA; as a reflection in changes in the diet (model 2); then $\mathrm{VO}_{2 \max }$, as a reflection of changes in physical activity (model 3 ); and body fat percentage (model 4). None of these regression parameters showed intercorrelations $>0.6$. HOMA-IR was ln-transformed to obtain a normal distribution. For regression analysis, the beta coefficients and their 95\% CIs are presented. Other data are expressed as means \pm SEM. A $p$ value $<0.05$ was considered statistically significant. Statistical analysis was performed using SPSS 10.0 for Macintosh (SPSS, Chicago, IL, USA).

\section{Results}

General lifestyle effects

After 1 year of lifestyle intervention, body weight and BMI were reduced, $\mathrm{VO}_{2 \max }$ was increased, and improvements were seen in 2-h glucose values, fasting insulin and HOMAIR values (Table 1). The lifestyle intervention was effective in increasing the intake of carbohydrates and fibre and reducing the intake of total fat and saturated fat, and concomitantly reduced the monounsaturated fat intake (Table 2), as previously reported in a smaller group in the same intervention study [22]. The reported intake of PUFA correlated well with PUFA in serum, both at baseline $(r=0.44, p<0.05)$ and after 1 year $(r=0.42, p<0.001)$. This indicates that the reported intakes of fatty acids were well recorded and estimated.

Serum fatty acid profile, glucose tolerance and insulin sensitivity

After 1 year of lifestyle intervention, no direct changes were observed in individual fatty acid fractions. However, changes in several specific fatty acid fractions of cholesteryl esters were related to changes in insulin resistance after 1 year (Table 3 ). Changes at 1 year in serum fractions of myristic (C14:0), palmitoleic acid (C16:1 $n$-7), $\gamma$-linolenic acid (C18:3n-6) and dihomo- $\gamma$-linolenic acid (C20:3 n-6) correlated positively with changes in HOMA-IR, whereas an inverse relationship was observed for oleic acid (C18:1 $n-9)$ and arachidonic acid (C20:4 n-6). Decreases in

Table 1 Subject characteristics at baseline and after 1 year of lifestyle intervention

\begin{tabular}{|c|c|c|c|c|c|c|c|}
\hline & \multicolumn{2}{|c|}{ Intervention } & \multicolumn{2}{|l|}{ Control } & \multicolumn{3}{|l|}{$p$ value } \\
\hline & Baseline & $\begin{array}{l}\text { After } \\
1 \text { year }\end{array}$ & Baseline & $\begin{array}{l}\text { After } \\
1 \text { year }\end{array}$ & Group & Time & Group $\times$ time \\
\hline$n$ (men/women) & $47(29 / 18)$ & $47(29 / 18)$ & $50(30 / 20)$ & $50(30 / 20)$ & & & \\
\hline Age (years) & $56.0 \pm 0.9$ & - & $58.5 \pm 1.0$ & - & 0.07 & - & - \\
\hline Body weight (kg) & $88.2 \pm 1.9$ & $85.4 \pm 1.8$ & $84.5 \pm 1.9$ & $83.8 \pm 1.8$ & 0.31 & $<0.01$ & $<0.01$ \\
\hline BMI $\left(\mathrm{kg} / \mathrm{m}^{2}\right)$ & $29.7 \pm 0.5$ & $28.8 \pm 0.5$ & $29.6 \pm 0.5$ & $29.4 \pm 0.5$ & 0.71 & $<0.01$ & 0.01 \\
\hline Body fat (\%) & $37.4 \pm 0.9$ & $35.8 \pm 0.9$ & $37.5 \pm 0.9$ & $36.8 \pm 0.9$ & 0.67 & $<0.01$ & 0.08 \\
\hline Waist (cm) & $104 \pm 1$ & $100 \pm 2$ & $104 \pm 1$ & $102 \pm 1$ & 0.66 & $<0.1$ & 0.06 \\
\hline Fasting glucose $(\mathrm{mmol} / \mathrm{l})$ & $6.1 \pm 0.1$ & $6.0 \pm 0.1$ & $5.8 \pm 0.1$ & $5.9 \pm 0.1$ & 0.17 & 0.70 & 0.05 \\
\hline 2-h glucose $(\mathrm{mmol} / \mathrm{l})$ & $9.0 \pm 0.3$ & $8.1 \pm 0.3$ & $8.5 \pm 0.3$ & $8.7 \pm 0.3$ & 0.91 & 0.10 & $<0.01$ \\
\hline $\mathrm{HbA}_{1 \mathrm{c}}(\%)$ & $6.0 \pm 0.1$ & $5.8 \pm 0.1$ & $5.9 \pm 0.1$ & $5.7 \pm 0.1$ & 0.26 & $<0.01$ & 0.12 \\
\hline Fasting insulin (mU/l) & $17.9 \pm 1.2$ & $15.5 \pm 1.1$ & $17.3 \pm 1.2$ & $17.4 \pm 1.1$ & 0.70 & 0.06 & 0.04 \\
\hline 2-h insulin $(\mathrm{mU} / \mathrm{l})$ & $93 \pm 12$ & $78 \pm 9$ & $103 \pm 11$ & $107 \pm 9$ & 0.16 & 0.31 & 0.06 \\
\hline HOMA-IR & $5.00 \pm 0.40$ & $4.22 \pm 0.35$ & $4.58 \pm 0.39$ & $4.67 \pm 0.34$ & 0.98 & 0.08 & 0.03 \\
\hline $\mathrm{VO}_{2 \max }\left(\mathrm{ml} \mathrm{O} \mathrm{kg} \mathrm{FFM}^{-1} \min ^{-1}\right)$ & $40.9 \pm 1.0$ & $43.3 \pm 1.1$ & $39.8 \pm 1.0$ & $39.9 \pm 1.0$ & 0.11 & $<0.01$ & 0.01 \\
\hline
\end{tabular}

Data are presented as means \pm SEM

FFM Fat-free mass 
Table 2 Dietary intake of macronutrients for the intervention and control group at baseline and after 1 year of a combined diet/exercise lifestyle intervention

\begin{tabular}{|c|c|c|c|c|c|c|c|}
\hline & \multicolumn{2}{|c|}{ Intervention } & \multicolumn{2}{|l|}{ Control } & \multicolumn{3}{|l|}{$p$ value } \\
\hline & Baseline & 1 year & Baseline & 1 year & Group & Time & Group $\times$ time \\
\hline$n$ (men/women) & $47(29 / 18)$ & $47(29 / 18)$ & $50(30 / 20)$ & $50(30 / 20)$ & & & \\
\hline Energy intake (MJ/day) & $9.2 \pm 0.4$ & $8.2 \pm 0.3$ & $8.6 \pm 0.4$ & $8.4 \pm 0.3$ & 0.63 & $<0.01$ & 0.07 \\
\hline Carbohydrate $(E \%)$ & $42 \pm 1$ & $47 \pm 1$ & $43 \pm 1$ & $44 \pm 1$ & 0.61 & $<0.01$ & $<0.01$ \\
\hline Fat $(E \%)$ & $35.9 \pm 0.9$ & $30.7 \pm 0.9$ & $35.3 \pm 0.9$ & $34.0 \pm 0.9$ & 0.21 & $<0.01$ & $<0.01$ \\
\hline SFA $(E \%)$ & $13.6 \pm 0.4$ & $11.3 \pm 0.5$ & $13.7 \pm 0.4$ & $13.0 \pm 0.4$ & 0.08 & $<0.01$ & 0.01 \\
\hline MUFA $(E \%)$ & $13.0 \pm 0.4$ & $10.9 \pm 0.4$ & $12.7 \pm 0.4$ & $12.0 \pm 0.4$ & 0.36 & $<0.01$ & 0.02 \\
\hline PUFA $(E \%)$ & $6.8 \pm 0.3$ & $6.3 \pm 0.3$ & $6.5 \pm 0.3$ & $6.5 \pm 0.3$ & 0.82 & 0.30 & 0.28 \\
\hline Cholesterol (mg/MJ) & $27 \pm 1$ & $22 \pm 2$ & $26 \pm 1$ & $25 \pm 1$ & 0.56 & $<0.01$ & 0.04 \\
\hline Protein $(E \%)$ & $16.5 \pm 0.5$ & $17.5 \pm 0.5$ & $16.0 \pm 0.5$ & $16.0 \pm 0.5$ & 0.07 & 0.15 & 0.21 \\
\hline Fibre $(\mathrm{g} / \mathrm{MJ})$ & $2.7 \pm 0.1$ & $3.3 \pm 0.1$ & $2.7 \pm 0.1$ & $2.8 \pm 0.1$ & 0.09 & $<0.01$ & $<0.01$ \\
\hline Alcohol (E\%) & $5.5 \pm 0.9$ & $4.7 \pm 0.9$ & $5.4 \pm 0.9$ & $5.4 \pm 0.9$ & 0.75 & 0.48 & 0.51 \\
\hline
\end{tabular}

Data are presented as means \pm SEM

estimated $\Delta 9$ - and $\Delta 6$-desaturase activities and an increase in estimated $\Delta 5$-desaturase activity at 1 year were related to a reduction in HOMA-IR values (Table 3).

To investigate whether the relationship between desaturase activity and insulin resistance was dependent on changes in lifestyle, we performed regression analysis and presented the standardised $\beta$-coefficients of the estimated desaturase activities after adjustment for changes in SFA intake, $\mathrm{VO}_{2 \max }$ and percentage body fat (Table 4). The results show that the changes in $\Delta 9$-and $\Delta 6$-desaturase were positively related to changes in HOMA-IR, whereas changes in $\Delta 5$-desaturase were negatively related. These relationships remained statistically significant after adjustment for changes in lifestyle factors and percentage body fat for all three desaturases. The $\beta$-coefficients of $\Delta 9$ - and $\Delta 6$-desaturase were reduced after the addition of $\mathrm{VO}_{2 \max }$ $(-13 \%$ and $-14 \%$, respectively; model 3$)$ and percentage body fat ( $-24 \%$ and $-4 \%$, respectively; model 4$)$ to the model, whereas the $\beta$-coefficient of $\Delta 5$-desaturase only changed after correction for percentage body fat $(-16 \%$; model 4$)$.

Since the correlation of a parameter with HOMA-IR can occasionally be fully explained by one component of the HOMA-IR, we explored the associations of fasting glucose and fasting insulin with different fatty acid variables. We

Table 3 Fatty acid fractions of serum cholesteryl esters at baseline, 1-year changes in fatty acid fractions and the relationships between changes in fatty acid fractions and lifestyle-induced changes in HOMA-IR

\begin{tabular}{|c|c|c|c|}
\hline & Baseline value & 1-year change & $\Delta$ HOMA-IR (ln) \\
\hline & Mean \pm SEM & Mean (range) & $r$ \\
\hline $14: 0$ & $0.84 \pm 0.02$ & $-0.013(-0.49$ to 0.55$)$ & $0.28 * *$ \\
\hline $16: 0$ & $11.7 \pm 0.1$ & $-0.005(-1.76$ to 1.60$)$ & -0.13 \\
\hline $16: 1 n-7$ & $3.4 \pm 0.1$ & $-0.06(-2.44$ to 2.46$)$ & $0.22 *$ \\
\hline $18: 0$ & $0.95 \pm 0.02$ & $0.01(-0.45$ to 0.26$)$ & -0.14 \\
\hline $18: 1 n-9$ & $16.9 \pm 0.2$ & $0.51(-3.84$ to 5.82$)$ & $-0.28 * *$ \\
\hline $18: 2 n-6$ & $52.7 \pm 0.5$ & $-0.49(-9.06$ to 5.46$)$ & +0.19 \\
\hline $18: 3 n-3$ & $0.61 \pm 0.01$ & $0.04(-0.28$ to 0.60$)$ & +0.13 \\
\hline $18: 3 n-6$ & $1.06 \pm 0.04$ & $0.04(-1.13$ to 1.59$)$ & $+0.32 * *$ \\
\hline $20: 3 n-6$ & $0.87 \pm 0.02$ & $-0.001(-0.57$ to 0.32$)$ & $+0.20 *$ \\
\hline $20: 4 n-6$ & $7.3 \pm 0.2$ & $-0.08(-3.22$ to 3.14$)$ & $-0.29 * *$ \\
\hline $20: 5 n-3$ & $1.11 \pm 0.06$ & $0.02(-2.98$ to 1.14$)$ & +0.14 \\
\hline $22: 6 n-3$ & $0.63 \pm 0.02$ & $0.00(-0.52$ to 0.36$)$ & -0.13 \\
\hline C20:4 n-6/C20:3 n-6 & $8.69 \pm 2.59$ & $-0.14(-8.92$ to 9.14$)$ & $-0.31 * *$ \\
\hline C18:3 n-6/C18:2 n-6 & $0.021 \pm 0.008$ & $0.001(-0.022$ to 0.032$)$ & $+0.28 * *$ \\
\hline $\mathrm{C} 16: 0 / \mathrm{C} 16: 1 n-7$ & $0.286 \pm 0.109$ & $-0.005(-0.19$ to 0.25$)$ & $+0.24 *$ \\
\hline
\end{tabular}

Fatty acid fractions of serum cholesteryl esters are expressed as percentage of total; $n=97$

$* p<0.05, * * p<0.01$ 
Table 4 Regression model for changes after 1 year: relationships between changes in HOMA-IR (ln) and changes in estimated desaturase activity

\begin{tabular}{|c|c|c|c|c|c|c|c|}
\hline & & $\begin{array}{l}\text { Std beta }(95 \% \mathrm{CI}) \\
\text { of } \Delta 9 \text {-desaturase activity }\end{array}$ & $\mathrm{a} R^{2}$ & $\begin{array}{l}\text { Std beta }(95 \% \mathrm{CI}) \\
\text { of } \Delta 6 \text {-desaturase activity }\end{array}$ & $\mathrm{a} R^{2}$ & $\begin{array}{l}\text { Std beta }(95 \% \mathrm{CI}) \\
\text { of } \Delta 5 \text {-desaturase activity }\end{array}$ & $\mathrm{a} R^{2}$ \\
\hline 1 & Desaturase & $0.234 *(0.032-0.438)$ & 0.04 & $0.278 * *(0.077-0.479)$ & 0.06 & $-0.307 * *(-0.501$ to -0.108$)$ & 0.08 \\
\hline \multirow[t]{2}{*}{2} & Desaturase & $0.251 *(0.055-0.448)$ & 0.10 & $0.274 * *(0.079-0.479)$ & 0.11 & $-0.296^{* *}(-0.484$ to -0.102$)$ & 0.13 \\
\hline & SFA intake & $0.271 * *(0.076-0.466)$ & & $0.252 *(0.059-0.437)$ & & $0.242 *(0.050-0.426)$ & \\
\hline \multirow[t]{3}{*}{3} & Desaturase & $0.218 *(0.028-0.406)$ & 0.18 & $0.237 *(0.050-0.426)$ & 0.19 & $-0.301 * *(-0.478$ to -0.118$)$ & 0.23 \\
\hline & SFA intake & $0.281 * *(0.094-0.468)$ & & $0.264 * *(0.085-0.451)$ & & $0.255^{* *}(0.077-0.434)$ & \\
\hline & $\mathrm{VO}_{2 \max }$ & $-0.295^{* *}(-0.492$ to -0.111$)$ & & $-0.289 * *(-0.470$ to -0.108$)$ & & $-0.324 * *(-0.504$ to -0.144$)$ & \\
\hline \multirow[t]{4}{*}{4} & Desaturase & $0.167 *(0.005-0.331)$ & 0.40 & $0.226^{* *}(0.068-0.386)$ & 0.43 & $-0.254 * *(-0.405$ to -0.097$)$ & 0.44 \\
\hline & SFA intake & $0.194 *(0.034-0.354)$ & & $0.178 *(0.025-0.331)$ & & $0.175 *(0.017-0.325)$ & \\
\hline & $\mathrm{VO}_{2 \max }$ & $-0.310^{* *}(-0.465$ to -0.155$)$ & & $-0.301 * *(-0.458$ to -0.144$)$ & & $-0.332 * *(-0.474$ to -0.178$)$ & \\
\hline & $\mathrm{BF} \%$ & $0.478 * *(0.315-0.635)$ & & $0.491^{* *}(0.333-0.649)$ & & $0.470 * *(0.317-0.629)$ & \\
\hline
\end{tabular}

Model 1: change in desaturase activity, adjusted for mean HOMA-IR; Model 2: + change in SFA intake (E\%); Model 3: + change in $\mathrm{VO}_{2 \max }(\mathrm{ml} \mathrm{O} / \mathrm{kg}$ fat-free mass); Model 4: + change in $\mathrm{BF} \%$; $n=97$

Std beta Standardised $\beta$-coefficient, $C I$ confidence interval; $a R^{2}$ adjusted R-square, Desaturase desaturase activity mentioned above

the column; $B F \%$, percentage body fat

${ }^{*} p<0.05$

$* * p<0.01$

found that analysis of changes in fasting glucose or insulin as the dependent variable revealed significant correlations with changes in $\Delta 9-, \Delta 6$-, and $\Delta 5$-desaturase indices. Pearson correlation coefficients for the relationships between fasting insulin and changes in $\Delta 9-, \Delta 6-$, and $\Delta 5$ desaturase were $r=0.151(p=0.067), r=0.196(p=0.016)$ and $r=-0.243(p=0.002)$, respectively. Pearson correlation coefficients for the associations between changes in fasting glucose and changes in $\Delta 9-, \Delta 6$-, and $\Delta 5$-desaturase were $r=0.199(p=0.045), r=0.287 \quad(p=0.003)$ and $r=-0.210$ $(p=0.033)$, respectively. These data reveal that no one specific component of the HOMA-IR (glucose or insulin) can explain the correlations with HOMA-IR. It appears that both impaired glucose and impaired insulin concentrations are relevant, indicating disturbances in the regulation of glucose metabolism. Since insulin resistance is most likely to be central to these impairments, we chose to present the data on HOMA-IR.

Interestingly, the relationships between changes in estimated desaturase enzyme activities and changes in HOMA-IR were modified by total fat intake. Regression analysis revealed that changes in $\Delta 9$ - and $\Delta 6$-desaturase contributed significantly to the HOMA-IR model in subjects with a total fat intake $<35.5 E \%$, and followed patterns similar to those observed in the group as a whole, whereas no significant association was found in subjects with a fat intake $>35.5 E \%$ (Fig. 1b,d). Also, in regression analysis of the total group, a relevant interaction between fat intake (below or above $35.5 \mathrm{E} \%$ ) and change in $\Delta 9$-desaturase activity was found $(p=0.13)$. The correlation between the change in $\Delta 5$-desaturase and insulin resistance was not affected by total fat intake (Fig. 1e,f).

\section{Discussion}

In this study, a strong relationship between the lifestyleinduced changes in insulin resistance and changes in cholesteryl ester fatty acid profile was found after 1 year. An improvement in insulin resistance was typically associated with reductions in myristic acid (C14:0), palmitoleic acid (C16:1 n-7), $\gamma$-linolenic acid (C18:3 n-6) and dihomo$\gamma$-linolenic acid (C20:3 n-6) fractions, and an increase in oleic acid (C18:1 n-9) and arachidonic acid (C20:4 n-6) fractions. This improvement was further characterised by a decrease in estimated $\Delta 9$-desaturase (SCD-1) and $\Delta 6$ desaturase activities and an increase in $\Delta 5$-desaturase activity.

This specific pattern of individual fatty acids observed (high myristic, palmitoleic, $\gamma$-linolenic and dihomo- $\gamma$ linolenic acid fractions and a low arachidonic acid fraction) is consistent with previous reports on insulin resistance [5, 9, 18, 30]. Furthermore, in agreement with the majority of previous studies, we found a positive relationship between changes in oleic acid fractions and changes in insulin sensitivity at 1 year. On the one hand, dietary studies have found that insulin sensitivity is inversely associated with oleic acid fractions $[18,30]$. On the other hand, exercise, assumed to be related to insulin sensitivity, is positively associated with high oleic acid fractions [19]. In addition, the replacement of dietary SFA by MUFA (predominantly oleic acid) improved insulin sensitivity [8], which supports the positive relationship observed between changes in serum oleic acid and insulin sensitivity in the present study.

The relationship between insulin sensitivity and $n-3$ PUFA is less clear. The present study shows no correlation between eicosapentaenoic acid (C20:5 n-3) or docosahex- 


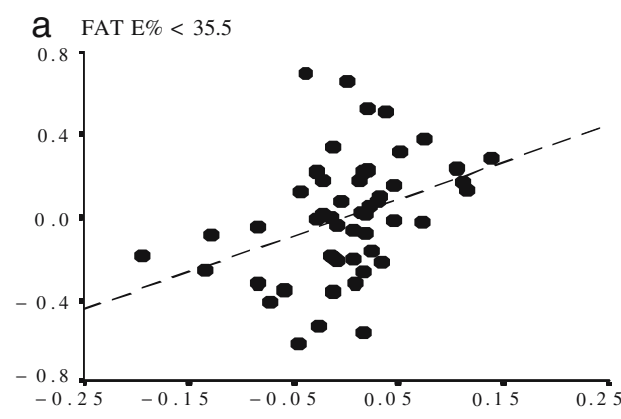

Change in $\Delta 9-$ desaturase

\section{C}

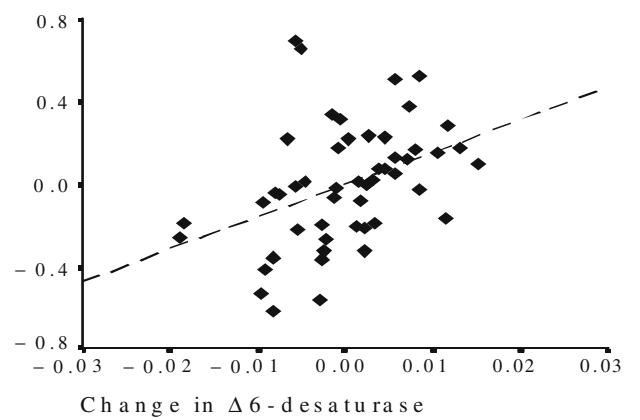

e

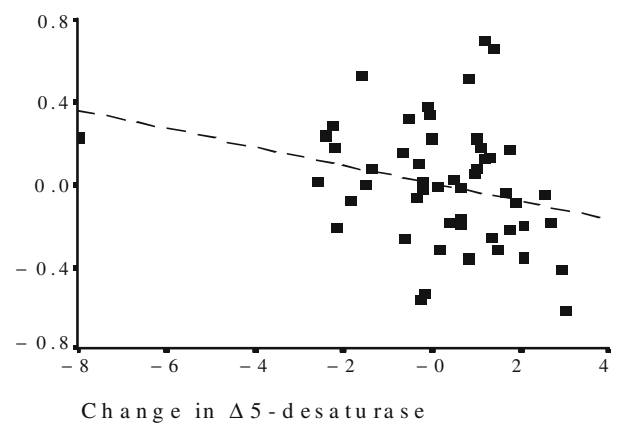

Fig. 1 Regression model for changes after 1 year: changes in HOMAIR $(\ln )$ related to changes in estimated $\Delta 9$-desaturase activity (a, b), $\Delta 6$-desaturase activity (c, d) and $\Delta 5$-desaturase activity $(\mathbf{e}, \mathbf{f})$ in subjects with a total dietary fat intake below a, c, e or above

aenoic acid (C22:6 n-3) and insulin resistance (Table 3). This is in accordance with other studies using other measures of insulin resistance, such as the euglycaemichyperinsulinaemic clamp or an IVGTT [8, 31-33].

The present study shows that changes in insulin resistance were inversely related to changes in $\Delta 5$ desaturase activity and positively related to changes in $\Delta 6$-desaturase and $\Delta 9$-desaturase activities. Desaturase enzymes may be important in the development of insulin resistance, since mice with a $\Delta 9$-desaturase deficiency are very insulin sensitive [13], and the $\Delta 9$-desaturase mRNA level in human skeletal muscle was positively related to the amount of triglyceride accumulation [15]. This accumula-

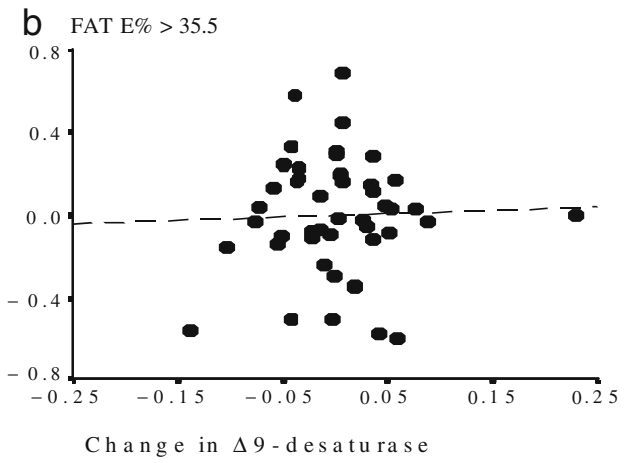

d
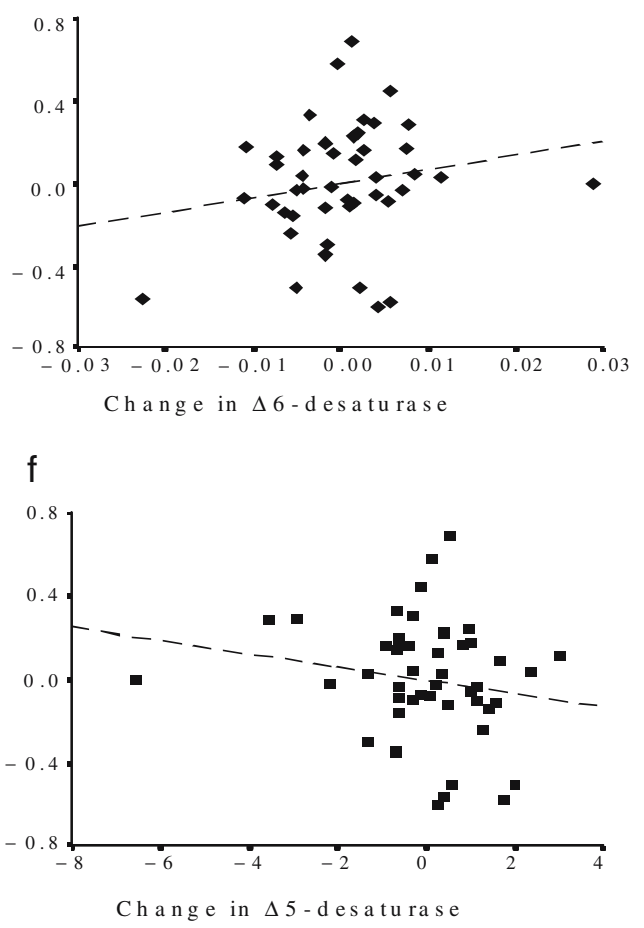

b, d, f median $(35.5 E \%)$. Partial correlations adjusted for mean HOMA-IR, change in SFA intake, change in $\mathrm{VO}_{2 \max }$ and change in percentage body fat (model 4). $p<0.01$ (a, c); $p<0.05$ (e, f)

tion of triglycerides in skeletal muscle is strongly associated with insulin resistance in sedentary subjects [34-36].

The associations between changes in $\Delta 9-, \Delta 6$ - and $\Delta 5$ desaturase activities and changes in insulin resistance in the present study were influenced by changes in saturated fat intake, $\mathrm{VO}_{2 \max }$ and/or percentage body fat, which suggests that desaturase activities are affected by lifestyle. The finding that lifestyle affects fatty acid profile is supported by studies in both rodents and humans. In mice, it was shown that a high-fat diet increased the $\Delta 9$-desaturase activity in the liver [37, 38]. Dietary intervention studies in humans have shown that replacing saturated dietary fat with unsaturated dietary fat reduces the estimated $\Delta 6$-desaturase 
and $\Delta 9$-desaturase activities, and increases the estimated $\Delta 5$-desaturase activity [16]. Furthermore, increased physical activity correlates with an increased estimated $\Delta 5$ desaturase activity in skeletal muscle phospholipids [20].

Part of the relationship between changes in insulin resistance and the changes in $\Delta 6$-desaturase and $\Delta 9$ desaturase activities may be a direct consequence of changes in lifestyle. The finding that these associations remained after adjustment for lifestyle factors (saturated fat intake, $\mathrm{VO}_{2 \max }$ and percentage body fat) indicates that changes in desaturase activities are also affected by endogenous factors. Many dietary, hormonal and environmental factors are involved in the regulation of $\Delta 9$ desaturase, and are likely to be involved in modulation of $\Delta 6$ - and $\Delta 5$-desaturase $[10,39]$. Therefore, we cannot exclude the possibility that insulin resistance may have had an effect on desaturase activities. Other factors that may be involved are changes in insulin [40] or glucose itself, changes in leptin concentration [41] or changes in fatty acid handling with different preferences for saturated or unsaturated fatty acids in lipolytic and oxidative processes [42, 43]. One challenge for future studies is to elucidate whether and how lifestyle factors can modify desaturase enzyme activities with consequences for the insulin-resistant state, and whether and how changes in metabolic profile, e.g. insulin resistance, may in turn affect desaturase enzyme activity.

A general hypothesis on the molecular background of the relationship between desaturase enzymes and insulin resistance is that desaturases can change the fatty acid composition of cell membranes, which influence membrane fluidity. This may result in changes in insulin receptor binding or affinity, membrane ion permeability and cell signalling [3]. Furthermore, $\Delta 9$-desaturase may produce precursors for compounds that have been associated with insulin resistance, such as ceramide [13]. The $\Delta 6$ - and $\Delta 5$ desaturases are involved in the synthesis of highly unsaturated fatty acids. These highly unsaturated fatty acids can act as ligands for transcription factors such as the peroxisome proliferator-activator receptors, hepatocyte nuclear factor 4 , nuclear factor $\mathrm{\kappa B}$ and sterol regulatory element binding protein, which interact with genes involved in lipogenesis and fatty acid oxidation [12].

In the present study, the relationships between changes in fatty acid profile and changes in insulin resistance were modified by total fat intake (Fig. 1). The association of changes in estimated $\Delta 9$ - and $\Delta 6$-desaturase activities with changes in insulin sensitivity were more pronounced in subjects with a lower total fat intake $(<35.5 \mathrm{E} \%)$. This has been reported previously in the Kuopio, Aarhus, Naples, Wollongong and Uppsala (KANWU) study, a large dietcontrolled study [8], which reported that the impact of fatty acid profile, reflecting dietary fat quality, on insulin sensitivity was mainly observed in subjects with a total fat intake below the median $(<37 E \%)$. This emphasises that both the quality and quantity of dietary fat intake are relevant in terms of insulin resistance. It also shows that a high fat intake may mask the potential relationship between fat quality and insulin resistance. The correlation between the change in $\Delta 5$-desaturase and insulin resistance was not affected by total fat intake (Fig. 1 and Table 4) or by physical activity (Table 4 ), which suggests that $\Delta 5$ desaturase activity may be influenced by environmental factors to a lesser degree than $\Delta 9$ - and $\Delta 6$-desaturase activities. A previous study examined the predictive effect of desaturase indices on the development of the metabolic syndrome and found that the relationships between $\Delta 9$ - and $\Delta 6$-desaturase and the development of the metabolic syndrome diminished after adjustment for BMI plus smoking habit plus physical activity, whereas the predictive value of $\Delta 5$-desaturase on the development of the metabolic syndrome was not affected after adjustment for BMI or physical activity [18].

In the present study, both insulin sensitivity and desaturase activity were measured indirectly. Nevertheless, the present data should be considered a clear indication of the relationship between insulin resistance and fatty acid profile, including the activity of desaturase enzymes, which deserves further investigation. Mechanistic studies using more direct measures, such as the hyperinsulinaemiceuglycaemic clamp and analysis of desaturase activity and mRNA expression in a variety of tissues, will be the next step in the elucidatation of the relationship between desaturase enzymes and lifestyle-induced changes in insulin sensitivity.

In summary, lifestyle-induced improvements in insulin sensitivity are independently explained by specific changes in the fatty acid profile of serum cholesteryl esters. Moreover, an increase in insulin sensitivity is associated with an increase in estimated $\Delta 5$-desaturase activity and a decrease in estimated $\Delta 6$ - and $\Delta 9$-desaturase activities. The associations between changes in $\Delta 9-, \Delta 6$ - and $\Delta 5$ desaturase activities and changes in insulin resistance remained significant after adjustment for changes in diet, $\mathrm{VO}_{2 \max }$ and/or body fat percentage. We conclude that lifestyle-induced changes in insulin sensitivity are partly related to changes in fatty acid profile, particularly changes in desaturase activities. The association between insulin resistance and desaturase activities is dependent on lifestyle, but also on changes in other, more endogenous factors that remain as yet unidentified.

Acknowledgements We would like to thank J. Stegen for the analytical measurements, and $\mathrm{H}$. Cremers for his excellent support for the analysis of fatty acid profiles. Furthermore, we would like to thank T. Hermans-Limpens, I. Nijs and M. van der Hulst for running the dietary intervention. This work was supported by grants from the 
Dutch Diabetes Research Foundation (DFN 98.901 and DFN 2000.00.020) and the Netherlands Organization for Scientific Research (ZonMW 940-35-034 and 2200.0139).

Duality of interest T. de Bruin is employed by GlaxoSmithKline, USA. The work in this paper was carried out while T. de Bruin was employed by the Academic Hospital Maastricht, The Netherlands.

\section{References}

1. Feskens EJ, van Dam RM (1999) Dietary fat and the etiology of type 2 diabetes: an epidemiological perspective. Nutr Metab Cardiovasc Dis 9:87-95

2. Hu FB, van Dam RM, Liu S (2001) Diet and risk of type II diabetes: the role of types of fat and carbohydrate. Diabetologia 44:805-817

3. Riccardi G, Giacco R, Rivellese AA (2004) Dietary fat, insulin sensitivity and the metabolic syndrome. Clin Nutr 23:447-456

4. Pelikanova T, Kohout M, Valek J, Base J, Kazdova L (1989) Insulin secretion and insulin action related to the serum phospholipid fatty acid pattern in healthy men. Metabolism 38:188-192

5. Vessby B, Tengblad S, Lithell H (1994) Insulin sensitivity is related to the fatty acid composition of serum lipids and skeletal muscle phospholipids in 70-year-old men. Diabetologia 37:1044-1050

6. Vessby B, Aro A, Skarfors E, Berglund L, Salminen I, Lithell $\mathrm{H}$ (1994) The risk to develop NIDDM is related to the fatty acid composition of the serum cholesterol esters. Diabetes 43: 1353-1357

7. Wang L, Folsom AR, Zheng ZJ, Pankow JS, Eckfeldt JH (2003) Plasma fatty acid composition and incidence of diabetes in middle-aged adults: the Atherosclerosis Risk in Communities (ARIC) Study. Am J Clin Nutr 78:91-98

8. Vessby B, Unsitupa M, Hermansen K et al (2001) Substituting dietary saturated for monounsaturated fat impairs insulin sensitivity in healthy men and women: The KANWU Study. Diabetologia 44:312-319

9. Summers LK, Fielding BA, Bradshaw HA et al (2002) Substituting dietary saturated fat with polyunsaturated fat changes abdominal fat distribution and improves insulin sensitivity. Diabetologia 45 : 369-377

10. Nakamura MT, Nara TY (2004) Structure, function, and dietary regulation of delta6, delta5, and delta9 desaturases. Annu Rev Nutr 24:345-376

11. Dobrzyn A, Ntambi JM (2004) The role of stearoyl-CoA desaturase in body weight regulation. Trends Cardiovasc Med 14:77-81

12. Jump DB, Clarke SD (1999) Regulation of gene expression by dietary fat. Annu Rev Nutr 19:63-90

13. Dobrzyn A, Dobrzyn P, Lee SH et al (2005) Stearoyl-CoA desaturase-1 deficiency reduces ceramide synthesis by downregulating serine palmitoyltransferase and increasing beta-oxidation in skeletal muscle. Am J Physiol Endocrinol Metab 288:E599-E607

14. Borkman M, Storlien LH, Pan DA, Jenkins AB, Chisholm DJ, Campbell LV (1993) The relation between insulin sensitivity and the fatty-acid composition of skeletal-muscle phospholipids. N Engl J Med 328:238-244

15. Hulver MW, Berggren JR, Carper MJ et al (2005) Elevated stearoyl-CoA desaturase-1 expression in skeletal muscle contributes to abnormal fatty acid partitioning in obese humans. Cell Metab 2:251-261

16. Vessby B, Gustafsson IB, Tengblad S, Boberg M, Andersson A (2002) Desaturation and elongation of fatty acids and insulin action. Ann N Y Acad Sci 967:183-195
17. Kelley DE, Goodpaster BH, Storlien L (2002) Muscle triglyceride and insulin resistance. Annu Rev Nutr 22:325-346

18. Warensjo E, Riserus U, Vessby B (2005) Fatty acid composition of serum lipids predicts the development of the metabolic syndrome in men. Diabetologia 48:1999-2005

19. Andersson A, Sjodin A, Olsson R, Vessby B (1998) Effects of physical exercise on phospholipid fatty acid composition in skeletal muscle. Am J Physiol 274:E432-E438

20. Andersson A, Sjodin A, Hedman A, Olsson R, Vessby B (2000) Fatty acid profile of skeletal muscle phospholipids in trained and untrained young men. Am J Physiol Endocrinol Metab 279:E744-E751

21. Mensink M, Blaak EE, Corpeleijn E, Saris WH, de Bruin TW, Feskens EJ (2003) Lifestyle intervention according to general recommendations improves glucose tolerance. Obes Res 11:1588-1596

22. Mensink M, Corpeleijn E, Feskens EJ et al (2003) Study on lifestyle-intervention and impaired glucose tolerance Maastricht (SLIM): design and screening results. Diabetes Res Clin Pract 61:49-58

23. World Health Organization (1999) Definition, diagnosis and classification of diabetes mellitus and its complications. Report of a WHO consultation part 1: diagnosis and classification of diabetes mellitus. WHO technical report series 1-59

24. Health Council of the Netherlands (1992) Dutch dietary reference intakes 1989. The Hague, Voorlichtingsbureau voor de Voeding (In Dutch)

25. American College of Sports Medicine Position Stand (1998) The recommended quantity and quality of exercise for developing and maintaining cardiorespiratory and muscular fitness, and flexibility in healthy adults. Med Sci Sports Exerc 30:975-991

26. Durnin JV, Womersley J (1974) Body fat assessed from total body density and its estimation from skinfold thickness: measurements on 481 men and women aged from 16 to 72 years. Br J Nutr 32:77-97

27. Matthews DR, Hosker JP, Rudenski AS, Naylor BA, Treacher DF, Turner RC (1985) Homeostasis model assessment: insulin resistance and beta-cell function from fasting plasma glucose and insulin concentrations in man. Diabetologia 28:412-419

28. Wallace TM, Levy JC, Matthews DR (2004) Use and abuse of HOMA modeling. Diabetes Care 27:1487-1495

29. Agren JJ, Julkunen A, Penttila I (1992) Rapid separation of serum lipids for fatty acid analysis by a single aminopropyl column. J Lipid Res 33:1871-1876

30. Vessby B (2000) Dietary fat and insulin action in humans. Br J Nutr 83(Suppl 1):S91-S96

31. Borkman M, Chisholm DJ, Furler SM et al (1989) Effects of fish oil supplementation on glucose and lipid metabolism in NIDDM. Diabetes 38:1314-1319

32. McManus RM, Jumpson J, Finegood DT, Clandinin MT, Ryan EA (1996) A comparison of the effects of $n-3$ fatty acids from linseed oil and fish oil in well-controlled type II diabetes. Diabetes Care 19:463-467

33. Rivellese AA, Maffettone A, Iovine C et al (1996) Long-term effects of fish oil on insulin resistance and plasma lipoproteins in NIDDM patients with hypertriglyceridemia. Diabetes Care 19:1207-1213

34. Pan DA, Lillioja S, Kriketos AD et al (1997) Skeletal muscle triglyceride levels are inversely related to insulin action. Diabetes 46:983-988

35. Krssak M, Falk Petersen K, Dresner A et al (1999) Intramyocellular lipid concentrations are correlated with insulin sensitivity in humans: a ${ }^{1} \mathrm{H}$ NMR spectroscopy study. Diabetologia 42:113-116

36. Schmitz-Peiffer C (2000) Signalling aspects of insulin resistance in skeletal muscle: mechanisms induced by lipid oversupply. Cell Signal 12:583-594 
37. Hu CC, Qing K, Chen Y (2004) Diet-induced changes in stearoylCoA desaturase 1 expression in obesity-prone and -resistant mice. Obes Res 12:1264-1270

38. Biddinger SB, Almind K, Miyazaki M, Kokkotou E, Ntambi JM, Kahn CR (2005) Effects of diet and genetic background on sterol regulatory element-binding protein-1c, stearoyl-CoA desaturase 1 , and the development of the metabolic syndrome. Diabetes 54:1314-1323

39. Ntambi JM, Miyazaki M (2004) Regulation of stearoyl-CoA desaturases and role in metabolism. Prog Lipid Res 43:91104
40. Brenner RR (2003) Hormonal modulation of delta6 and delta5 desaturases: case of diabetes. Prostaglandins Leukot Essent Fatty Acids 68:151-162

41. Cohen P, Friedman JM (2004) Leptin and the control of metabolism: role for stearoyl-CoA desaturase-1 (SCD-1). J Nutr $134: 2455 \mathrm{~S}-2463 \mathrm{~S}$

42. Blaak EE (2004) Basic disturbances in skeletal muscle fatty acid metabolism in obesity and type 2 diabetes mellitus. Proc Nutr Soc 63:323-330

43. Raclot T, Oudart H (1999) Selectivity of fatty acids on lipid metabolism and gene expression. Proc Nutr Soc 58:633-646 